\title{
ON THE HOMOTOPY GROUPS OF THE EXCEPTIONAL LIE GROUPS $\left({ }^{1}\right)$
}

\author{
BY \\ P. G. KUMPEL, JR.
}

1. Introduction. Throughout this paper the symbols $G_{2}, F_{4}, E_{6}, E_{7}$ denote compact simply connected forms of these exceptional Lie groups. $\operatorname{Spin}(n)$ is the universal covering group of $\operatorname{SO}(n)$. There are inclusions $G_{2} \subset \operatorname{Spin} 8 \subset F_{4} \subset E_{6}$ given by Jacobson [13]. We also use the inclusion $E_{6} \subset E_{7}$ described using representations given by Tits [16] for the Lie algebras of these groups. $Q_{p}$ denotes the ring of rational numbers whose denominators are powers of the prime $p$. The following isomorphisms are obtained for $j \geqq 0$ :

$$
\begin{aligned}
\pi_{j}(\operatorname{Spin} 8) \otimes Q_{3} & \approx\left(\pi_{j}\left(G_{2}\right) \oplus \pi_{j}\left(\operatorname{Spin} 8 / G_{2}\right)\right) \otimes Q_{3}, \\
\pi_{j+1}\left(F_{4} / \text { Spin } 8\right) \otimes Q_{3} & \approx\left(\pi_{j+1}\left(F_{4} / G_{2}\right) \oplus \pi_{j}\left(\text { Spin } 8 / G_{2}\right)\right) \otimes Q_{3}, \\
\pi_{j}\left(E_{6}\right) \otimes Q_{2} & \approx\left(\pi_{j}\left(F_{4}\right) \oplus \pi_{j}\left(E_{6} / F_{4}\right)\right) \otimes Q_{2}, \\
\pi_{j+1}\left(E_{7} / E_{6}\right) \otimes Q_{2} & \approx\left(\pi_{j+1}\left(E_{7} / F_{4}\right) \oplus \pi_{j}\left(E_{6} / F_{4}\right)\right) \otimes Q_{2} .
\end{aligned}
$$

(1.1) was stated without proof by Harris [8]. The proofs of (1.1), (1.2), (1.3), (1.4) are given in $\S \S 3,4,5,6$ respectively. $\$ 2$ contains a discussion of results of Jacobson and Tits as they relate to the inclusions mentioned earlier. Actually, in $\S 5$, the proof of the following theorem, which implies (1.3), is given:

THEOREM 1.5. Let $G$ be a compact, connected, simply connected Lie group, $\sigma: G \rightarrow G$ an automorphism of period $2, K$ the identity component of the fixed point set of $\sigma$. Assume the map $H^{*}(G ; R) \rightarrow H^{*}(K ; R)$ induced by inclusion is an epimorphism. Then for all $i$ the sequence $0 \rightarrow \pi_{i}(K) \rightarrow \pi_{i}(G) \rightarrow \pi_{i}(G / K) \rightarrow 0$ is exact and split when tensored with $Q_{2}$. The splitting is given by the map $q: G / K \rightarrow G, q(g K)=g \sigma(g)^{-1}, g \in G$.

TheOREM 1.5 applies to the following pairs $(G, K)$ : $(\mathrm{SU}(2 n+1), \mathrm{SO}(2 n+1))$, $(\operatorname{SU}(2 n), \operatorname{Sp}(n)),(\operatorname{Spin}(2 n), \operatorname{Spin}(2 n-1))$ and $\left(E_{6}, F_{4}\right)$. In each of the first three cases, the result was given by Harris [8], and the splitting for $\left(E_{6}, F_{4}\right)$ was given by Harris provided the primes 2 and 3 are neglected. The proof of this theorem is

Received by the editors November 3, 1964.

(1) This research was supported in part by NSF Grant 24026. 
based on a suggestion of Harris (see [8]). The idea for using Poincaré duality is from the work of Araki [1]. In [8], Harris obtains a similar result, except that primes $p$ for which $G$ has $p$-torsion must also be neglected. In [1], Araki obtains the conclusion of 1.5 under the hypothesis that $\operatorname{rank} G=\operatorname{rank} K+\operatorname{rank} G / K$. Araki's hypothesis implies that $H^{*}(G ; R) \rightarrow H^{*}(K ; R)$ is an epimorphism. It is to be emphasized that the proof of 1.5 does not depend on the classification of symmetric spaces.

In $§ 7$, it is shown that by combining (1.4) with similar results of Harris, and using the classification of symmetric spaces, the following theorem is obtained:

THEOREM 1.6. Let $G, K, \sigma$ satisfy the hypotheses of Theorem 1.5. In addition, assume $G$ is simple. Then there exists a simple group $L$, containing $G$, such that the sequence $0 \rightarrow \pi_{j+1}(L / G) \rightarrow \pi_{j+1}(L / K) \rightarrow \pi_{j}(G / K) \rightarrow 0$ is exact and split when tensored with $Q_{2}$. The splitting is given by the Bott suspension map (see [6]) $\pi_{j}(G / K) \rightarrow \pi_{j+1}(L / G)$. Moreover, rank $L-\operatorname{rank} G \leqq 1$ and either $L / G$ or $L / M$ is a symmetric space, where $M$ is locally isomorphic to $G \times S^{1}$.

Theorem 1.6 applies to the following triples $(L, G, K):(\mathrm{SO}(4 n), \mathrm{SU}(2 n)$, $\mathrm{Sp}(n)),(\operatorname{Sp}(2 n+1), \mathrm{SU}(2 n+1), \mathrm{SO}(2 n+1)), \operatorname{Spin}(2 n+1),(\operatorname{Spin}(2 n), \operatorname{Spin}(2 n-1))$ and $\left(E_{7}, E_{6}, F_{4}\right)$. The results in the first three cases were obtained by Harris [8].

The results of this paper are contained in the author's doctoral dissertation. The author wishes to thank Professor Bruno Harris of Brown University for suggesting these problems and for his advice and criticism throughout the preparation of the manuscript. These results were announced in the Notices of the American Mathematical Society, abstracts 63T-53, January 1963, and 64T-173, February 1964.

2. Descriptions of the groups and algebras. Representations of $G_{2}, F_{4}$ and $E_{6}$ are given by Jacobson [13]. Denote by $\mathscr{K}$ the nonassociative algebra of Cayley numbers. This is an 8-dimensional real vector space. It can be described by naming generators $1, i, j, l$ subject to the relations $i^{2}=j^{2}=l^{2}=-1, i j=-j i, i l=-l i$, $j l=-l j$. A basis is given by $1, i, j, i j, l, i l, j l,(i j) l$. The subalgebra generated by $1, i, j$ is the algebra of quaternions. Every Cayley number is uniquely expressible in the form $a+b l, a, b$ quaternions. For quaternion $a, a=a_{0}+a_{1} i+a_{2} j+a_{3} i j, a_{i}$ real, define $\bar{a}=a_{0}-a_{1} i-a_{2} j-a_{3} i j$, and for $x=a+b l \in \mathscr{K}$ define $\bar{x}=\bar{a}-b l$. The norm of $x \in \mathscr{K}$ is given by $N(x)=x \bar{x}=\bar{x} x$ and is a real number. The associated bilinear form $N(x, y)=(x \bar{y}+y \bar{x}) / 2$ is nondegenerate and the basis mentioned above is orthonormal. Also

$$
N(x y)=N(x) N(y), \quad x, y \in \mathscr{K} .
$$

Let $M_{3}^{8}$ be the exceptional Jordan algebra of 3 by 3 hermitian matrices with coefficients in $\mathscr{K}$; an element $A \in M_{3}^{8}$ is a 3 by 3 Cayley matrix with $A^{\text {tr }}=A$. Multiplication in $M_{3}^{8}$ is given by $A \cdot B=(A B+B A) / 2$, where juxtaposition denotes usual matrix multiplication. Thus, if $A \in M_{3}^{8}$, we may write 


$$
A=\left[\begin{array}{lll}
\alpha_{1} & a & b \\
\bar{a} & \alpha_{2} & c \\
\bar{b} & \bar{c} & \alpha_{3}
\end{array}\right], \quad \alpha_{i} \text { real, } \quad a, b, c \in \mathscr{K}
$$

Clearly $M_{3}^{8}$ is a 27 -dimensional real vector space.

Define $\varepsilon_{1}=\operatorname{diag}(1,0,0), \varepsilon_{2}=\operatorname{diag}(0,1,0), \varepsilon_{3}=\operatorname{diag}(0,0,1)$, and for $a \in \mathscr{K}$, define $a_{i j}, i \neq j, i, j=1,2,3$, to be the 3 by 3 matrix whose only nonzero entries are $a$ in the $(i, j)$ position and $\bar{a}$ in the $(j, i)$ position. Then (2.2) may be written

$$
A=\sum_{i=1}^{3} \alpha_{i} \varepsilon_{i}+a_{12}+b_{13}+c_{23}
$$

The trace of an element of $\mathscr{K}$ is twice its real part, i.e. $T(a)=a+\bar{a}, a \in \mathscr{K}$. The trace and norm of an element $A \in M_{3}^{8}$, written as in (2.3), are defined, respectively, by

$$
\begin{aligned}
& T(A)=\sum_{i=1}^{3} \alpha_{i}, \\
& N(A)=\alpha_{1} \alpha_{2} \alpha_{3}+T(a(c \bar{b}))-\alpha_{1} N(c)-\alpha_{2} N(\bar{b})-\alpha_{3} N(a) .
\end{aligned}
$$

Multiplication in $M_{3}^{8}$ is characterized by the formulas:

$$
\begin{aligned}
\varepsilon_{i} \cdot a_{i j} & =\frac{1}{2} a_{i j}=a_{i j} \cdot \varepsilon_{j}, \\
a_{i j}^{2} & =N(a)\left(\varepsilon_{i}+\varepsilon_{j}\right), \\
2 a_{i j} \cdot b_{j k} & =(a b)_{i k}, \quad i, j, k \text { unequal, }
\end{aligned}
$$

and by the fact that $\varepsilon_{i}$ are orthogonal idempotents.

Jacobson $[13, \mathrm{III}]$ has shown that the group of norm-preserving linear transformation of $M_{3}^{8}$ is a compact form of $E_{6}$, and $[13, \mathrm{II}]$ that the group of all automorphisms of the Jordan algebra $M_{3}^{8}$ is the compact $F_{4}$. In the same series of papers, it is shown that

$$
\left\{t \in F_{4}: t\left(\varepsilon_{i}\right)=\varepsilon_{i}, i=1,2,3\right\} \approx \operatorname{Spin} 8,
$$

$$
\left\{t \in F_{4}: t\left(1_{i j}\right)=1_{i j}, i \neq j, i, j=1,2,3, \quad t\left(\varepsilon_{i}\right)=\varepsilon_{i}, i=1,2,3\right\} \approx G_{2} .
$$

Moreover, if $\varepsilon$ is a primitive idempotent, that is, an idempotent which is not the sum of two orthogonal idempotents (equivalently, $T(\varepsilon)=1$ ), then

$$
\left\{t \in F_{4}: t(\varepsilon)=\varepsilon\right\} \approx \text { Spin } 9 .
$$

We now proceed to describe the compact Lie algebra $\mathscr{E}_{7}$ and its subalgebras $\mathscr{E}_{6}$ and $\mathscr{F}_{4}$ (compact forms) using the work of J. Tits [16].

Let $\mathscr{Y}$ be the complex simple 3-dimensional Lie algebra. $\mathscr{Y}$ has basis $\{e, f, h\}$ 
over the complex numbers, $C$, with multiplication given by $[e h]=2 e,[f h]=-2 f$, $[e f]=h$. The compact real form of $\mathscr{Y}$, henceforth denoted by $Y$, has real basis $\{i h, e+f, i(e-f)\}, i=(-1)^{1 / 2}$. If $($,$) denotes the Killing form of \mathscr{Y}$, one computes that $(h, h)=8,(e, f)=-4,(e, h)=(f, h)=(e, e)=(f, f)=0$. Following [16], the compact Lie algebra $\mathscr{E}_{7}$ is

$$
\mathscr{E}_{7}=\mathscr{D}\left(M_{3}^{8}\right) \oplus\left(Y \otimes M_{3}^{8}\right)
$$

where $\mathscr{D}\left(M_{3}^{8}\right)$ is the Lie algebra of derivations of $M_{3}^{8}, \mathscr{D}\left(M_{3}^{8}\right)=\mathscr{F}_{4}$, and where the multiplication in $\mathscr{E}_{7}$ is given by

$$
\begin{aligned}
{[d, y \otimes a] } & =y \otimes d(a), \\
{\left[y \otimes a, y^{\prime} \otimes a^{\prime}\right] } & =\frac{1}{2}\left(y, y^{\prime}\right)\left\langle a, a^{\prime}\right\rangle+\left[y y^{\prime}\right] \otimes a a^{\prime},
\end{aligned}
$$

for $y, y^{\prime} \in Y, d \in \mathscr{D}\left(M_{3}^{8}\right), a, a^{\prime} \in M_{3}^{8}$. The symbol $\left\langle a, a^{\prime}\right\rangle$ denotes the inner derivation of $M_{3}^{8}$ defined by

$$
\left\langle a, a^{\prime}\right\rangle\left(a^{\prime \prime}\right)=a\left(a^{\prime} a^{\prime \prime}\right)-a^{\prime}\left(a a^{\prime \prime}\right), \quad a^{\prime \prime} \in M_{3}^{8} .
$$

We have noted that $\mathscr{D}\left(M_{3}^{8}\right)=\mathscr{F}_{4}$ (see paragraph preceding (2.9)). Tits has shown that the subalgebra

$$
\mathscr{D}\left(M_{3}^{8}\right) \oplus \frac{i h}{2} \otimes M_{3}^{8}(0)
$$

is isomorphic to the compact $\mathscr{E}_{6}$. Here $M_{3}^{8}(0)$ denotes the set of elements of trace zero in $M_{3}^{8}$. Observe that $\frac{1}{2}(i h / 2, i h / 2)=-1$, so the multiplication described in (2.14) applied to an element of (2.16) is

$$
\left[\frac{i h}{2} \otimes a, \frac{i h}{2} \otimes a^{\prime}\right]=-\left\langle a, a^{\prime}\right\rangle .
$$

If $R_{a}: M_{3}^{8} \rightarrow M_{3}^{8}$ denotes right multiplication by $a \in M_{3}^{8}$, then $\left\langle a, a^{\prime}\right\rangle=-\left[R_{a} R_{a^{\prime}}\right]$, for

$$
\begin{aligned}
-\left[R_{a}, R_{a}^{\prime}\right]\left(a^{\prime \prime}\right) & =-\left(a^{\prime \prime} R_{a} R_{a^{\prime}}-a^{\prime \prime} R_{a^{\prime}} R_{a}\right) \\
& =-\left(\left(a^{\prime \prime} a\right) a^{\prime}-\left(a^{\prime \prime} a^{\prime}\right) a\right) \\
& =a\left(a^{\prime} a^{\prime \prime}\right)-a^{\prime}\left(a a^{\prime \prime}\right)=\left\langle a, a^{\prime}\right\rangle\left(a^{\prime \prime}\right) .
\end{aligned}
$$

Jacobson $\left[12\right.$, p.145] has described the Lie algebra $\mathscr{E}_{6}$ by

$$
\mathscr{E}_{6}=\mathscr{D}\left(M_{3}^{8}\right) \oplus\left\{R_{a}: a \in M_{3}^{8}(0)\right\} .
$$

The representations (2.16) and (2.18) are isomorphic under the identification of $i h / 2 \otimes a$ with $R_{a}$, because of (2.17) and the remarks following it. 
Of course, $\mathscr{D}\left(M_{3}^{8}\right) \oplus\left(i h / 2 \otimes M_{3}^{8}\right)$ is the direct sum of $\mathscr{E}_{6}$ with a 1-dimensional subalgebra.

Later, in $\S 6$, we will see that these inclusions $\mathscr{F}_{4} \subset \mathscr{E}_{6} \subset \mathscr{E}_{7}$ give rise to inclusions $F_{4} \subset E_{6} \subset E_{7}$ of the simply connected Lie groups.

3. The fibration $G_{2} \rightarrow \operatorname{Spin} 8 \rightarrow \operatorname{Spin} 8 / G_{2}$. In this section we prove (1.1). Recall, from $\S 2$, that $F_{4}$ is the group of all automorphisms of $M_{3}^{8}$, and that

$$
\begin{aligned}
\operatorname{Spin} 8 & =\left\{\tau \in F_{4}: \tau\left(\varepsilon_{i}\right)=\varepsilon_{i}, i=1,2,3\right\}, \\
G_{2} & =\left\{\tau \in \operatorname{Spin} 8: \tau\left(1_{i j}\right)=1_{i j}, i \neq j, i, j=1,2,3\right\} .
\end{aligned}
$$

Let $\eta: M_{3}^{8} \rightarrow M_{3}^{8}$ be defined by $\eta\left(\varepsilon_{i}\right)=\varepsilon_{i+1}, \eta\left(a_{i j}\right)=a_{i+1, j+1}, i \neq j, i, j=1,2,3$ (addition of subscripts modulo 3 ), and extend linearly. Define $\sigma: \operatorname{Spin} 8 \rightarrow \operatorname{Spin} 8$ by $\sigma(\tau)=\eta \tau \eta^{-1}, \tau \in$ Spin 8 . Then $\sigma$ is an automorphism of Spin 8 , and since $\eta^{3}=$ identity of $M_{3}^{8}, \sigma$ has order 3. Moreover, the fixed set of $\sigma$ is exactly the subgroup $G_{2}$.

Define a map $q:$ Spin $8 / G_{2} \rightarrow$ Spin 8 by $q\left(\tau G_{2}\right)=\tau \sigma(\tau)^{-1}, \tau \in$ Spin 8 . Let $p:$ Spin $8 \rightarrow \operatorname{Spin} 8 / G_{2}$ be the natural projection and $j: G_{2} \rightarrow$ Spin 8 the inclusion.

THEOREM 3.1. The exact homotopy sequence of the fiber space

$$
G_{2} \rightarrow \operatorname{Spin} 8 \rightarrow \operatorname{Spin} 8 / G_{2}
$$

is split when tensored by $Q_{3}$. The splitting is given by the map

$$
q: \text { Spin } 8 / G_{2} \rightarrow \operatorname{Spin} 8
$$

and we obtain $\mathscr{C}_{3}$-isomorphisms

$$
\pi_{j}(\operatorname{Spin} 8) \approx \pi_{j}\left(G_{2}\right) \oplus \pi_{j}\left(\operatorname{Spin} 8 / G_{2}\right), \quad j \geqq 0 .
$$

Proof. We compute the composition

$$
p^{*} q^{*}: \pi_{j}\left(\operatorname{Spin} 8 / G_{2}\right) \rightarrow \pi_{j}\left(\operatorname{Spin} 8 / G_{2}\right) .
$$

To do this, we use the fact that there is a homeomorphism Spin $8 / G_{2} \rightarrow S^{7} \times S^{7}$. Here we consider $S^{7}$ as the unit 7 -sphere of Cayley numbers of norm 1 . The homeomorphism is defined by sending $\tau G_{2}, \tau \in \operatorname{Spin} 8$ into the pair $(u, v)$, where $u, v$ are Cayley numbers determined by $\tau\left(1_{12}\right)=u_{12}, \tau\left(1_{13}\right)=v_{13}$. See Jacobson [13, II, p. 93].

Since $\sigma$ leaves $G_{2}$ pointwise fixed, it induces a map

$$
\sigma: \operatorname{Spin} 8 / G_{2} \rightarrow \operatorname{Spin} 8 / G_{2} \text {. }
$$

We identify Spin $8 / G_{2}$ with $S^{7} \times S^{7}$ as above and compute $\sigma$. In fact, if $\tau \in \operatorname{Spin} 8$ is such that $\tau G_{2}$ corresponds to the pair $(u, v)$ in $S^{7} \times S^{7}$, then

$$
\tau\left(1_{23}\right)=\tau\left(2\left(1_{21} \cdot 1_{13}\right)\right)=2 \tau\left(1_{12}\right) \cdot \tau\left(1_{13}\right)=2 u_{12} v_{13}=(\bar{u} v)_{23} .
$$


Hence

and by similar calculation

$$
\begin{aligned}
\sigma(\tau)\left(1_{12}\right) & =\eta \tau \eta^{-1}\left(1_{12}\right) \\
& =\eta \tau\left(1_{31}\right) \\
& =\eta \tau\left(1_{13}\right) \\
& =\eta\left(v_{13}\right)=v_{21}=\bar{v}_{12},
\end{aligned}
$$

$$
\sigma(\tau)\left(1_{13}\right)=(\overrightarrow{v u})_{13}
$$

Thus if $G_{2}$ corresponds to $(u, v) \in S^{7} \times S^{7}, \sigma(\tau) G_{2}$ corresponds to the pair $(\bar{v}, \bar{v} u) \in S^{7} \times S^{7}$, and $\sigma: S^{7} \times S^{7} \rightarrow S^{7} \times S^{7}$ given by

$$
\sigma(u, v)=(\bar{v}, \bar{v} u)
$$

Since $\bar{v}$ is the multiplicative inverse of $v$, if we identify $\pi_{j}\left(S^{7} \times S^{7}\right)$ with $\pi_{j}\left(S^{7}\right) \oplus \pi_{j}\left(S^{7}\right)$, it follows that the induced map $\sigma_{*}: \pi_{j}\left(S^{7} \times S^{7}\right) \rightarrow \pi_{j}\left(S^{7} \times S^{7}\right)$ is given by

$$
\sigma_{*}((\alpha, \beta))=(-\beta,-\beta+\alpha), \quad \alpha, \beta \in \pi_{j}\left(S^{7}\right) .
$$

We now compute $p_{*} q_{*}$. From the definition, if $\tau \in \operatorname{Spin} 8$,

$$
p q\left(\tau G_{2}\right)=\tau \sigma(\tau)^{-1} G_{2} .
$$

Let $T=p q$. To simplify the notation, let $K=\operatorname{Spin} 8$, and $H=G_{2}$. We have a multiplication

$$
\mu: K / H \times K / H \rightarrow K / H
$$

defined by $\mu\left(k_{1} H, k_{2} H\right)=k_{1} \sigma\left(k_{1}\right)^{-1} k_{2} H$. One easily verifies that

(a) $\mu(\varepsilon H, k H)=k H, \varepsilon$ the identity of $K, k \in K$,

(b) $\mu(k H, \sigma(k H))=k H$,

(c) $T(k H)=\mu(k H, \varepsilon H)$.

Identifying $\pi_{j}(K / H \times K / H)$ with $\pi_{j}(K / H) \oplus \pi_{j}(K / H)$ we obtain

(a) $\mu_{*}(0, a)=a$,

(b) $\mu_{*}\left(a, \sigma_{*}(a)\right)=a$,

(c) $T_{*}(a)=\mu_{*}(a, 0), a \in \pi_{j}(K / H)$.

Therefore,

$$
\begin{aligned}
T^{*}(a) & =\mu_{*}(a, 0)=\mu_{*}\left(a, \sigma_{*}(a)-\sigma_{*}(a)\right) \\
& =\mu_{*}\left(\left(a, \sigma_{*}(a)\right)-\left(0, \sigma_{*}(a)\right)\right. \\
& =\mu_{*}\left(a, \sigma_{*}(a)\right)-\mu_{*}\left(0, \sigma_{*}(a)\right) \\
& =a-\sigma_{*}(a), a \in \pi_{j}(K / H) .
\end{aligned}
$$

Therefore if $\alpha, \beta \in \pi_{j}\left(S^{7}\right)$, we have 


$$
\begin{aligned}
T_{*}((\alpha, \beta)) & =(\alpha, \beta)-\sigma_{*}(\alpha, \beta) \\
& =(\alpha, \beta)-(-\beta,-\beta+\alpha)=(\alpha+\beta, 2 \beta-\alpha) .
\end{aligned}
$$

To complete the proof of Theorem 3.1, tensor the exact homotopy sequence of the fiber space $G_{2} \rightarrow$ Spin $8 \rightarrow \operatorname{Spin} 8 / G_{2}$ with $Q_{3}$. Exactness is preserved, since $Q_{3}$ has no torsion. Since division by 3 is now permissible, we may define the map

$$
S: \pi_{i}\left(S^{7} \times S^{7}\right) \otimes Q_{3} \rightarrow \pi_{i}\left(S^{7} \times S^{7}\right) \otimes Q_{3}
$$

by $S(\gamma, \delta)=((2 \gamma-\delta) / 3,(\gamma+\delta) / 3), \gamma, \delta \in \pi_{i}\left(S_{7}\right)$. Then

$$
T_{*} S(\alpha, \beta)=T_{*}((2 \gamma-\delta) / 3,(\gamma+\delta) / 3)=(\gamma, \delta),
$$

and

$$
S T_{*}(\alpha, \beta)=S(\alpha+\beta, 2 \beta-\alpha)=((2 \alpha+2 \beta-2 \beta+\alpha) / 2,3 \beta / 3)=(\alpha, \beta) .
$$

Hence $T_{*}$ is an automorphism after tensoring by $Q_{3}$. Thus the sequence splits, and 3.1 is proved.

4. The fibration Spin $8 / G_{2} \rightarrow F_{4} / G_{2} \rightarrow F_{4} /$ Spin 8 . In this section it is shown that the exact homotopy sequence of the fiber space Spin $8 / G_{2} \rightarrow F_{4} / G_{2} \rightarrow F_{4} /$ Spin 8 is split when tensored by $Q_{3}$, thereby proving (1.2). We retain the notation of the preceding sections, and we continue to describe these groups in terms of $M_{3}^{\mathbf{8}}$.

We begin by considering the set of all 3 by 3 matrices with Cayley coefficients, together with addition and ordinary matrix multiplication. This is a nonassociative algebra $\mathscr{A}$ over the real numbers. If $A$ is a 3 by 3 real nonsingular matrix, the mapping $\mathscr{A} \rightarrow \mathscr{A}$ defined by $B \rightarrow A B A^{-1}, B \in \mathscr{A}$, is an automorphism of the algebra $\mathscr{A}$. The product $A B A^{-1}$ is unambiguous since $A$ has real coefficients. If $\tau$ is an automorphism of the Cayley numbers, $\tau$ leaves the subalgebra of real numbers pointwise fixed. Hence if $B=\left(b_{i j}\right) \in \mathscr{A}$, the map $B \rightarrow \tau(B)=\left(\tau\left(b_{i j}\right)\right)$ is an automorphism of $\mathscr{A}$ and if $A$ has real coefficients, $\tau\left(A B A^{-1}\right)=A \tau(B) A^{-1}$. Also note that if $A \in O(3), B \in M_{3}^{8}$ then $A B A^{-1} \in M_{3}^{8}$, and the map $B \rightarrow A B A^{-1}$ is an automorphism of the Jordan algebra $M_{3}^{8}$.

Let $\tau \in G_{2}$. Then there is an automorphism $f$ of the Cayley numbers such that

$$
\left(\sum_{i=1}^{3} \alpha_{i} e_{i}+a_{12}+b_{23}+c_{13}\right)=\sum_{i=1}^{3} \alpha_{i} e_{i}+f(a)_{12}+f(b)_{23}+f(c)_{13}
$$

(see Jacobson [13,II]). Thus, if $B \in M_{3}^{8}, B=\left(b_{i j}\right)$, then $\tau(B)=\left(f\left(b_{i j}\right)\right)$, so $\tau$ is of the form described above. Hence we may state the

Lemma 4.1. If $A \in O(3), B \in M_{3}^{8}$, then $A B A^{-1} \in M_{3}^{8}$ and $\tau\left(A B A^{-1}\right)=A \tau(B) A^{-1}$. 
Proposition 4.2. There is a 1-parameter subgroup $\psi$ of $F_{4}$ such that

(a) $\psi(1)=\eta$, i.e., $\psi(1) x \psi(1)^{-1}=\sigma(x), x \in \operatorname{Spin} 8$,

(b) $\psi(t) \tau=\tau \psi(t), \tau \in G_{2}, t \in R$.

Proof. First note that $\eta$ is given by $\eta(B)=A B A^{-1}, B \in M_{3}^{8}$ where

$$
A=\left[\begin{array}{lll}
0 & 0 & 1 \\
1 & 0 & 0 \\
0 & 1 & 0
\end{array}\right] \in \operatorname{SO}(3)
$$

There is a 1-parameter subgroup $\phi(t)$ of $\mathrm{SO}(3)$ such that $\phi(1)=A$. (Every point of a compact connected Lie group lies on a 1-parameter subgroup.) Define $\psi(t): M_{3}^{8} \rightarrow M_{3}^{8}$ by $\psi(t)(B)=\phi(t) B \phi(t)^{-1}$. From the preceding remarks, $\psi$ is a subgroup of $F_{4}$. Since $\psi(1)(B)=A B A^{-1}, \psi(1)=n$ and (a) is satisfied. If $\tau \in G_{2}$, then for all $B \in M_{3}^{8}, \quad \psi(t) \tau(B)=\phi(t) \tau(B) \phi(t)^{-1}$ which equals, by (4.1), $\tau\left(\phi(t) B \phi(t)^{-1}\right)=\tau \psi(t)(B)$. Hence, for all $\tau \in G_{2}$, and all real $t, \psi(t) \tau=\tau \psi(t)$, and (b) is satisfied.

Having proved the existence of a 1-parameter subgroup $\psi$ of $F_{4}$ such that

(a) $\psi(1) \tau \psi(1)^{-1}=\sigma(\tau), \tau \in \operatorname{Spin} 8$, and

(b) $\psi(t) \tau=\tau \psi(t), \tau \in G_{2}, t$ real,

we can construct a map $F: s\left(\operatorname{Spin} 8 / G_{2}\right) \rightarrow F_{4} /$ Spin 8 . Here $s\left(\operatorname{Spin} 8 / G_{2}\right)$ denotes the suspension of Spin $8 / G_{2}$, i.e., the space obtained from (Spin $\left.8 / G_{2}\right) \times[0,1]$ by collapsing (Spin $8 / G_{2}$ ) $\times 1$ and (Spin $8 / G_{2}$ ) $\times 0$ to points. $F$ is defined by $F\left(\tau G_{2}, t\right)=\tau \psi(t)$ Spin 8. Following Harris [8], if $E$ denotes the composition $\pi_{j}\left(\operatorname{Spin} 8 / G_{2}\right) \rightarrow \pi_{j+1}\left(s\left(\operatorname{Spin} 8 / G_{2}\right)\right) \rightarrow \rightarrow^{F^{*}} \pi_{j+1}\left(F_{4} /\right.$ Spin 8$)$, where the first map is ordinary suspension, and if $\partial$ is the boundary operator of the homotopy sequence of the fibration, $\partial: \pi_{j+1}\left(F_{4} / \operatorname{Spin} 8\right) \rightarrow \pi_{j}\left(\operatorname{Spin} 8 / G_{2}\right)$, then $T_{*}=\partial E$, where, as in the preceding section, $T=p q$.

But in $\S 3$, we saw that after tensoring with $Q_{3}, T_{*}$ is an automorphism of $\pi_{j}\left(\right.$ Spin $\left.8 / G_{2}\right)$, hence $E$ provides an inverse to $\partial$ and the exact homotopy sequence

$$
\cdots \rightarrow \pi_{j+1}\left(F_{4} / G_{2}\right) \rightarrow \pi_{j+1}\left(F_{4} / \operatorname{Spin} 8\right) \stackrel{\partial}{\rightarrow} \pi_{j}\left(\operatorname{Spin} 8 / G_{2}\right) \rightarrow \cdots
$$

is split when tensored with $Q_{3}$.

Finally, we remark that $F_{4}$ is, in a sense, the smallest group containing Spin 8 in which $\sigma$ becomes inner (in the sense of (4.2)). Evidently, if $G$ is a Lie group. and Spin $8 \subset G \subset F_{4}$, then $G$ is contained in a maximal subgroup of maximal rank of $F_{4}$. These subgroups are known by [5] to be $\operatorname{SU}(2) \times \operatorname{Sp}(3)$. Spin 9 and $\mathrm{SU}(3) \times \mathrm{SU}(3)$. The first and last are impossible for dimension reasons, and it is not difficult to check directly that $\sigma$ does not become inner in any representation of Spin 9 of the form (2.11).

5. The fibration $F_{4} \rightarrow E_{6} \rightarrow E_{6} / F_{4}$. Throughout this section, let $G$ denote a compact, connected, simply connected Lie group, $\sigma: G \rightarrow G$ an automorphism of 
order $2, K$ the identity component of the fixed point set of $\sigma$. We will give a proof of Theorem 1.5, and we show that this theorem applies to the pair $\left(E_{6}, F_{4}\right)$. For the proof of (1.5) we use certain results of Harris [8] which we summarize here.

THEOREM 5.1 (HARris). Let $G, \sigma, K$ be as above. Assume $H^{*}(G ; R) \rightarrow H^{*}(K ; R)$ is an epimorphism. Then primitive generators $x_{i}$ may be chosen for $H^{*}(G ; R)$. so that $\sigma^{*}\left(x_{i}\right)= \pm x_{i}$. Let $U$ be the subalgebra generated by the $x_{i}$ left fixed by $\sigma^{*}$, and let $V$ be the subalgebra generated by the other $x_{i}$ 's. Then:

(a) $H^{*}(G ; R) \approx U \otimes V$ as algebra,

(b) if $i: K \rightarrow G$ is inclusion, $i^{*}$ maps $U$ isomorphically onto $H^{*}(K ; R)$ and is zero on the positive degree elements of $V$,

(c) if $q: G / K \rightarrow G$ is the map $q(g K)=g \sigma(g)^{-1}$ then $q^{*}$ maps $V$ isomorphically onto $H^{*}(G / K ; R)$ and is zero on the positive degree elements of $U$,

(d) if $l: G \rightarrow G / K$ is projection, then im $l^{*}=V$ and $l^{*}$ is $1-1$; moreover $l^{*} q^{*}: H^{*}(G ; R) \rightarrow H^{*}(G ; R)$ is given by $l^{*} q^{*}(x)=x-\sigma^{*}(x), x$ primitive,

(e) $H^{*}(G / K ; R)$ has generators $y_{1}, \cdots, y_{t}$ such that $\sigma^{*}\left(y_{i}\right)=-y_{i}$ and $q^{*} l^{*}\left(y_{i}\right)=2 y_{i}, i=1, \cdots, t$.

Using (5.1), we proceed to show that the map $l q: G / K \rightarrow G / K$ is an isomorphism in homology with coefficients $Q_{2}$. Once this is accomplished, an application of the J. H. C. Whitehead theorem (see Serre $[14$, p. 276]), yields that

$$
l_{*} q_{*}: \pi_{j}(G / K) \rightarrow \pi_{j}(G / K)
$$

is a $\mathscr{C}_{2}$-isomorphism and hence $q_{*}$ gives the splitting described in (1.5).

Proof of (1.5). From (5.1.e), $H^{*}(G / K ; R)=\Lambda\left(y_{1}, \cdots, y_{t}\right)$, an exterior algebra on generators $y_{i}$ of odd degree such that

and

$$
\sigma^{*}\left(y_{i}\right)=-y_{i}, i=1, \cdots, t,
$$

$$
q^{*} l^{*}\left(y_{1} \cdots y_{t}\right)=2^{t} y_{1} \cdots y_{t} .
$$

Applying Poincaré duality to the simply-connected compact orientable manifold $G / K$, we obtain $H^{n}(G / K ; Z) \approx H_{0}(G / K ; Z) \approx Z$.

Let $\alpha$ be a generator of $H^{n}(G / K ; Z)$. Then $\alpha \otimes 1$ generates

$$
H^{n}(G / K ; R) \approx H^{n}(G / K ; Z) \otimes R .
$$

Let $q^{*} l^{*}(\alpha)=d \alpha, d \in Z$. Then if $I: R \rightarrow R$ is the identity map,

$$
\left(q^{*} l^{*} \otimes I\right)(\alpha \otimes 1)=d(\alpha \otimes 1) .
$$

But we know that $\left(q^{*} l^{*} \otimes I\right)\left(y_{1} \cdots y_{t}\right)=2^{t} y_{1} \cdots y_{t}$ and $\alpha \otimes 1=r y_{1} \cdots y_{t}$, some $r \in: R$, hence $q^{*} l^{*}(\alpha \otimes 1)=q^{*} l^{*}\left(r y_{1} \cdots y_{t}\right)=r 2^{t} y_{1} \cdots y_{t}=2^{t} \alpha \otimes 1$. Thus, $q^{*} l^{*}(\alpha)=2^{t} \alpha$.

Next consider $q^{*} l^{*}: H^{*}\left(G / K ; Q_{2}\right) \rightarrow H^{*}\left(G / K ; Q_{2}\right)$. Since division by 2 is possible, $q^{*} l^{*}$ is an automorphism in dimension $n$. 
Using the fact that real homology and cohomology are dual vector spaces, the above arguments hold for homology as well, and we obtain that

$$
l_{*} q_{*}: H_{n}\left(G / K ; Q_{2}\right) \rightarrow H_{n}\left(G / K ; Q_{2}\right)
$$

is an automorphism.

Let $u_{n}$ be a generator of $H_{n}\left(G / K ; Q_{2}\right)$. The Poincaré duality isomorphism $H^{i}\left(G / K ; Q_{2}\right) \rightarrow H_{n-i}\left(G / K ; Q_{2}\right)$ is given by $x \rightarrow u_{n} \cap x$. We show that $l_{*} q_{*}$ is an automorphism with coefficients $Q_{2}$.

Let $f=l q$. Recall the formula $f_{*}(a) \cap b=f_{*}\left(a \cap f_{*}(b)\right), a \in H_{*}\left(G / K ; Q_{2}\right)$, $b \in H^{*}\left(G / K ; Q_{2}\right)$. (See Hilton-Wylie $\left[10\right.$ p. 155].) We have $f^{*}\left(u_{n}\right)=2^{t} u_{n}$. Take $a=2^{-t} u_{n}$ in the formula. Then

i.e.,

$$
2^{-t} f_{*}\left(u_{n}\right) \cap b=2^{-t} f_{*}\left(u_{n} \cap f^{*}(b)\right)
$$

$$
u_{n} \cap b=2^{-t} f_{*}\left(u_{n} \cap f^{*}(b)\right) .
$$

From Poincaré duality, $u_{n} \cap b$ (and hence $\left.2^{t} u_{n} \cap b\right)$ ranges over $H_{*}\left(G / K ; Q_{2}\right)$ as $b$ takes on values in $H^{*}\left(G / K ; Q_{2}\right)$. Therefore $f_{*}$ is onto. It is known that a map of a finitely generated module over a Noetherian ring onto itself is necessarily 1-1. Hence $f_{*}$ is an automorphism. An application of the J. H.C. Whitehead theorem completes the proof of (1.5).

Next we show that (1.5) applies to the pair $\left(E_{6}, F_{4}\right)$ and hence that (1.3) holds. To do this we establish the following

Proposition 5.2. Let $G$ be a compact, connected, simple Lie group, $A(G)$ the automorphism group of $G, I(G)$ the subgroup of inner automorphisms. If

$$
A(G) / I(G) \approx Z_{2}
$$

and $\sigma \in A(G)$ is of order 2 and not inner, and if the exterior algebra $H^{*}(G ; R)$ has generators $x_{2 k_{i}-1}$, at least one $k_{i}$ odd, then

(a) $\sigma^{*}\left(x_{2 k_{i}-1}\right)=x_{2 k_{i}-1}$, if $k_{i}$ even.

(b) $\sigma^{*}\left(x_{2 k_{i}-1}\right)=-x_{2 k_{i}-1}$, if $k_{i}$ odd .

Proof. Let $B_{G}$ be the classifying space of $G$. Then $H^{*}\left(B_{G} ; R\right)$ is a polynomial algebra on generators $y_{i}, \operatorname{deg} y_{i}=2 k_{i}$, which suspend to the $x_{i}$. Let $T$ be a maximal torus of $G$, and let $\alpha \in I(G)$ such that $\alpha \circ \sigma: T \rightarrow T$. Since $\alpha^{*}$ is the identity on cohomology, we obtain induced maps $\sigma^{*}: H^{*}\left(B_{G} ; R\right) \rightarrow H^{*}\left(B_{G} ; R\right)$ and $\sigma^{*}: H^{*}\left(B_{T} ; R\right) \rightarrow H^{*}\left(B_{T} ; R\right)$. There is an inclusion $B_{T} \subset B_{G}$ which induces a monomorphism $H^{*}\left(B_{G} ; R\right) \rightarrow H^{*}\left(B_{T} ; R\right)$. But $H^{*}\left(B_{T} ; R\right)$ is a polynomial algebra on generators $z_{1}, \cdots, z_{l}(l=\operatorname{rank} G), \operatorname{deg} z_{i}=2$. Hence the generators of $H^{*}\left(B_{G} ; R\right)$ are homogeneous polynomials $P_{j}$ of degree $k_{j}$ in the $z$ 's.

Since $\sigma$ has order 2, we may assume that $\sigma^{*}\left(z_{i}\right)= \pm z_{i}, i=1, \cdots, l$. Consider the map $\gamma: T \rightarrow T, \gamma(t)=t^{-1}, t \in T$. Let $\mathscr{G}$ be the complexification of the Lie algebra 
of $G$. Then the Lie algebra $\mathscr{T}$ of $T$ (complexified) is a Cartan subalgebra of $\mathscr{G}$, and the map induced by $\gamma$ on $\mathscr{T}$ is $\gamma(h)=-h, h \in \mathscr{T}$.

Jacobson [12, p. 127] shows that the automorphism $\gamma: \mathscr{T} \rightarrow \mathscr{T}$ extends to an automorphism of order 2 of $\mathscr{G}$. Thus, passing to the compact Lie groups, the automorphism $\gamma: T \rightarrow T$ extends to an automorphism $\gamma$ of order $2, \gamma: G \rightarrow G$.

On $H^{*}\left(B_{T} ; R\right), \gamma^{*}\left(z_{i}\right)=-z_{\imath}, i=1, \cdots, l$. Hence on $H^{*}\left(B_{G} ; R\right), \gamma^{*} P_{j}=-P_{j}$ if and only if $k_{j}$ is odd. But there is an odd $k_{j}$, hence $\gamma$ is not the identity on $H^{*}(G ; R)$, hence $\gamma$ is not inner. Thus by the hypothesis, $\gamma=\tau \circ \sigma, \tau \in I(G)$. Hence $\gamma^{*}=\sigma^{*}$, so if $k_{j}$ is odd, $\sigma^{*}\left(x_{2 k_{j}-1}\right)=\gamma^{*}\left(x_{2 k_{j}-1}\right)$, and if $k_{j}$ is even,

This proves (5.2).

$$
\sigma^{*}\left(x_{2 k_{j}-1}\right)=x_{2 k_{j}-1} \text {. }
$$

Finally we point out that by Theorem 4 , Jacobson $[12$, p. 281$], E_{6}$ has only one outer automorphism (up to inner automorphisms), thus $E_{6}$ satisfies the hypotheses of (5.2). But $H^{*}\left(E_{6} ; R\right)=\Lambda\left(x_{3}, x_{9}, x_{11}, x_{15}, x_{17}, x_{23}\right)$ (see [4]), so if $\sigma$ is the involution of $E_{6}$ having $F_{4}$ as fixed point set, $\sigma^{*}\left(x_{9}\right)=-x_{9}$ and $\sigma^{*}\left(x_{17}\right)=-x_{17}$ since $9=4 \cdot 2+1$ and $17=4 \cdot 4+1$.

The following result of Harris [8] completes the proof.

Proposition 5.3 (HARRIS). If $G$ is a compact, connected, simply connected Lie group, $\sigma: G \rightarrow G$ an automorphism of order $2, K$ the identity component of the fixed point set of $\sigma$, we may write $H^{*}(G ; R)=U \otimes V$, where $U$ and $V$ are subalgebras such that $\sigma^{*}$ is the identity on $U$ and $\sigma^{*}$ multipltes the generators of $V$ by -1 . Moreover, if $V$ contains a nonzero element whose degree equals the dimension of $G / K$, then the map $H^{*}(G ; R) \rightarrow H^{*}(K ; R)$ induced by inclusion is an epimorphism.

Recall that $\sigma^{*}\left(x_{9}\right)=-x_{9}$ and $\sigma^{*}\left(x_{17}\right)=-x_{17}, x_{9}, x_{17}$ generators of $H^{*}\left(E_{6} ; R\right)$. Taking $U=\Lambda\left(x_{3}, x_{11}, x_{15}, x_{23}\right)$ and $V=\Lambda\left(x_{9}, x_{17}\right)$ and noting that $\operatorname{dim} x_{9} x_{17}=26=\operatorname{dim} E_{6} / F_{4}$, an application of (5.3) shows that $\left(E_{6}, F_{4}\right)$ satisfies the hypotheses of (1.5).

6. The fibration $E_{6} / F_{4} \rightarrow E_{7} / F_{4} \rightarrow E_{7} / E_{6}$. The purpose of this section is to establish (1.4). To do this we show that the homotopy exact sequence of the fibration $E_{6} / F_{4} \rightarrow E_{7} / F_{4} \rightarrow E_{7} / E_{6}$ is split when tensored with $Q_{2}$. The splitting is given by the Bott suspension map $E: \pi_{j}\left(E_{6} / F_{4}\right) \rightarrow \pi_{j+1}\left(E_{7} / E_{6}\right)$. We begin with some general remarks.

Let $K \subset G \subset L$ be compact connected Lie groups. Let $\sigma$ be an automorphism of period 2 of $G$ such that $K$ is the identity component of the fixed point set of $\sigma$. Harris [8] has shown that the Bott suspension map $E: \pi_{j}(G / K) \rightarrow \pi_{j+1}(L / G)$ may be constructed as follows: Let $v$ be a 1-parameter subgroup of $L$ such that (i) $v(1) g v(1)^{-1}=\sigma(g), g \in G$, (ii) $v(t) k=k v(t), k \in K, t \in R$. Let

$$
s: \pi_{j}(G / K) \rightarrow \pi_{j+1}(s(G / K))
$$


be the ordinary suspension. Define $F: s(G / K) \rightarrow L / G$ by $F(g K, t)=g v(t) G$, $g \in G, t \in R$. Then $E$ is the composition $F_{*} s: \pi_{j}(G / K) \rightarrow \pi_{j+1}(L / G)$. It is also shown in [8] that if $l: G \rightarrow G / K$ is the projection and $q: G / K \rightarrow G$ is the map $q(g K)=g \sigma(g)^{-1}, g \in G$, then the composition $l_{*} q_{*}: \pi_{j}(G / K) \rightarrow \pi_{j}(G / K)$ is the same as the composition $\partial E: \pi_{j}(G / K) \rightarrow \pi_{j}(G / K)$, $\partial$ the boundary map, $\partial: \pi_{j+1}(L / G) \rightarrow \pi_{j}(G / K)$, in the homotopy exact sequence of the fibration $G / K \rightarrow L / K \rightarrow L / G$. Thus $E$ serves as an inverse to $\partial$ in the homotopy sequence of $G / K \rightarrow L / K \rightarrow L / G$ if and only if $q_{*}$ serves as an inverse to $l_{*}$ in the homotopy sequence of $K \rightarrow G \rightarrow G / K$.

Since we have shown in $\S 5$ that $q_{*}$ gives a splitting of the homotopy sequence $F_{4} \rightarrow E_{6} \rightarrow E_{6} / F_{4}$, when tensored with $Q_{2}$, the above remarks make it clear that (1.4) will be proved if we can establish

Proposition 6.1. There exists a 1-parameter subgroup $v$ of $E_{7}$ such that (i) $v(1) g v(1)^{-1}=\sigma(g), g \in E_{6}$, where $\sigma$ is the automorphism of $E_{6}$ having $F_{4}$ as fixed point set, and (ii) $v(t) k=k v(t), k \in F_{4}, t \in R$.

The proof of the existence of such a $v$ proceeds as follows. Let $\mathscr{E}_{7}$ be the com pact Lie algebra described by (2.12), $\mathscr{E}_{6}$ the subalgebra given by (2.16) and $\mathscr{F}_{4}$ the subalgebra of $\mathscr{E}_{6}$ consisting of all derivations of $M_{3}^{8}$. Then $\mathscr{F}_{4} \subset \mathscr{E}_{6} \subset \mathscr{E}_{7}$ are all compact real forms. There is an automorphism $\sigma: \mathscr{E}_{6} \rightarrow \mathscr{E}_{6}$ defined by

$$
\sigma\left(d+\frac{i h}{2} \otimes a\right)=d-\frac{i h}{2} \otimes a, \quad d \in \mathscr{F}_{4}, a \in M_{3}^{8}(0) .
$$

The fixed set of $\sigma$ is $\mathscr{F}_{4}$, and $\sigma$ has period 2. We will define an automorphism $v: \mathscr{E}_{7} \rightarrow \mathscr{E}_{7}, v=\exp$ ad $x, x$ a certain element of $\mathscr{E}_{7}$, such that $v$ restricted to $\mathscr{E}_{6}$ is $\sigma$. We will show that $x$ may be chosen such that $\left[x \mathscr{F}_{4}\right]=0$. The exponential map Exp: $\mathscr{E}_{7} \rightarrow E_{7}$ will be used to transfer these data to the simply connected Lie groups $F_{4} \subset E_{6} \subset E_{7}$ (we will establish these inclusions later). Taking $v(t)$ $=\operatorname{Exp} t x, t \in R$, we will obtain the desired one-parameter subgroup. (i) is satisfied since $v \mid \mathscr{E}_{6}=\sigma$, hence the inner automorphism $g \rightarrow(\operatorname{Exp} x) g(\operatorname{Exp} x)^{-1}, g \in E_{7}$, coincides with $\sigma$ on $E_{6}$, and (ii) follows from $\left[x \mathscr{F}_{4}\right]=0$. We proceed with the details.

Proposition 6.2. Let $x=((e+f) \pi / 2) \otimes I \in Y \otimes M_{3}^{8} \subset \mathscr{E}_{7}, I$ the identity matrix. Then

(i) $\left[x \mathscr{F}_{4}\right]=0$ and

(ii) $\sigma$ is the restriction of the automorphism exp ad $x$ of $\mathscr{E}_{7}$ to the subalgebra $\mathscr{E}_{6}$.

Proof of 6.2. If $\left.\left.d \in \mathscr{F}_{4},[x d]=[((e+f) \pi / 2)) \otimes I, d\right]=-((e+f) \pi / 2)\right) \otimes d(I)$ by (2.13), and $d(I)=0$ since $d$ is a derivation. Hence (i) is established. To prove (ii), recall from $\S 2$, that

and that

$$
\begin{aligned}
{[i h, e+f] } & =-2 i(e-f), \\
{[i(e-f), e+f] } & =2 i h
\end{aligned}
$$




$$
\begin{aligned}
(i h, e+f) & =0, \\
(i(e-f), e+f) & =0,
\end{aligned}
$$

hence if $a \in M_{3}^{8}$, using formula (2.14) we obtain

$$
\begin{aligned}
(\operatorname{ad} x)^{2 k-1}(i h \otimes a) & =(-1)^{k} \pi^{2 k-1} i(e-f) \otimes a, \\
(\operatorname{ad} x)^{2 k}(i h \otimes a) & =(-1)^{k} \pi^{2 k} i h \otimes a .
\end{aligned}
$$

Therefore

$$
\begin{aligned}
(\exp \operatorname{ad} x)\left(\frac{i h}{2} \otimes a\right)= & \frac{i h}{2}\left(1-\frac{\pi^{2}}{2 !}+\frac{\pi^{4}}{4 !}-\cdots\right) \otimes a \\
& -\frac{i(e-f)}{2}\left(\pi-\frac{\pi^{3}}{3 !}+\frac{\pi^{5}}{5 !}-\cdots\right) \otimes a \\
= & -\frac{i h}{2} \otimes a .
\end{aligned}
$$

Since $\left[x \mathscr{F}_{4}\right]=0,(\exp$ ad $x) d=d, d \in \mathscr{F}_{4}$. Hence exp ad $x=\sigma$ and (ii) is proved, and this completes the proof of 6.2 .

Next we establish inclusions $F_{4} \subset E_{6} \subset E_{7}$ of the simply connected forms.

Let $E_{7}^{\prime}$ denote the adjoint group of $\mathscr{E}_{7}$ and $E_{6}^{\prime}$ the connected subgroup of $E_{7}^{\prime}$ corresponding to the subalgebra $\operatorname{ad}_{\mathscr{E}_{7}}\left(\mathscr{E}_{6}\right)$ of ad $\mathscr{E}_{7}($ ihe adjoint Lie algebra). We have the commutative diagram:

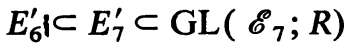

$$
\begin{aligned}
& \uparrow \uparrow \uparrow \exp \\
& \operatorname{ad}_{\mathscr{E}_{7}}\left(\mathscr{E}_{6}\right) \subset \operatorname{ad} \mathscr{E}_{7} \subset \operatorname{gl}\left(\mathscr{E}_{7} ; R\right) . \\
& \approx \uparrow \uparrow \\
& \mathscr{E}_{6} \subset \mathscr{E}_{7}
\end{aligned}
$$

Proposition 6.3. $E_{6}^{\prime}$ is the simply connected form of this group.

Proof of 6.3. It suffices to show that the center of $E_{6}^{\prime}$ contains a nontrivial element, since the center of the simply connected $E_{6}$ is $Z_{3}$. Let

$$
z=\frac{i h}{2} \otimes \operatorname{diag}(\lambda, \lambda,-2 \lambda), \quad \lambda=4 \pi / 3 \text {. }
$$

Then $z \in \mathscr{E}_{6}$. We will show that $\exp$ ad $z$ is a nontrivial element in the center of $E_{6}$. Let $Y^{\prime} \subset Y$ be the subspace spanned by $e+f, i(e-f)$. We first compute the eigenvalues of ad $z$ acting on $\mathscr{E}_{7}$. To do this, consider the complexification of $\mathscr{E}_{7}$. Then ad $z$ acting on $\mathscr{E}_{7} \otimes C$ has the complexified form of the subspaces

$$
\mathscr{D}\left(M_{3}^{8}\right) \oplus\left((i h / 2) \otimes M_{3}^{8}\right), \quad e \otimes M_{3}^{8}, \quad f \otimes M_{3}^{8}
$$

as invariant subspaces. For from (2.13), (2.14) and (2.17), 


$$
\begin{aligned}
(\operatorname{ad} z)(d+(i h / 2) \otimes a) & =(i h / 2) \otimes d(y)-\langle a, y\rangle, \\
(\operatorname{ad} z)(e \otimes a) & =[e, i h / 2] \otimes a y=i e \otimes a y, \\
(\operatorname{ad} z)(f \otimes a) & =[f, i h / 2] \otimes a y=-i f \otimes a y,
\end{aligned}
$$

where $y=\operatorname{diag}(\lambda, \lambda,-2 \lambda), d \in \mathscr{F}_{4}, a \in M_{3}{ }^{8}$. We propose to show

LEMmA 6.5. The eigenvalues of exp ad $z$ acting on $\mathscr{E}_{7} \otimes C$ are as follows:

(a) on $\left(\mathscr{D}\left(M_{3}^{8}\right) \oplus(i h / 2) \otimes M_{3}^{8}\right) \otimes C$, all eigenvalues are 1 ,

(b) on $\left(e \otimes M_{3}^{8}\right) \otimes C$, all eigenvalues are $\exp 4 \pi i / 3$,

(c) on $\left(f \otimes M_{3}^{8}\right) \otimes C$, all eigenvalues are $\exp 2 \pi i / 3$. Hence exp ad $z$ acts as the identity on $\left.\mathscr{D}\left(M_{3}^{8}\right) \oplus(\mathrm{ih} / 2) \otimes M_{3}^{8}\right) \otimes C$, as multiplication by exp $4 \pi i / 3$ on $\left(e \otimes M_{3}^{8}\right) \otimes C$ and as multiplication by $\exp 2 \pi i / 3$ on $\left(f \otimes M_{3}^{8}\right) \otimes C$.

Proof of 6.5. If $\mu$ is an eigenvalue of ad $z$ acting on $\mathscr{E}_{7} \otimes C$, then $\mu$ corresponds to an eigenvector in one of the invariant subspaces (6.4). If $(\mathrm{ad} z)(d+(i h / 2) \otimes m)$ $=\mu(d+(i h / 2) \otimes m), \quad d \in \mathscr{F}_{4}, \quad m \in M_{3}^{8}$, then $(i h / 2) \otimes d(y)-\langle m, y\rangle$ $=\mu(d+(i h / 2) \otimes m)$ so $d(y)=\mu m$ and $-\langle m, y\rangle=d$, from which we obtain $-\langle m, y\rangle(y)=\mu d(y)=\mu^{2} m$.

Thus $-m(y y)+y(m y)=\mu^{2} m$. But $y y=\lambda^{2}\left(\varepsilon_{1}+\varepsilon_{2}+4 \varepsilon_{3}\right)$, and if $m$ is written in the form of (2.3),

$$
m=\sum_{i=1}^{3} \alpha_{i} \varepsilon_{i}+a_{12}+b_{13}+c_{23}, \quad \alpha_{i} \text { real, } a, b, c \text { Cayley, }
$$

then

$$
m y=\lambda\left(\alpha_{1} \varepsilon_{1}+\alpha_{2} \varepsilon_{2}-2 \alpha_{3} \varepsilon_{3}+a_{12}-\frac{1}{2} b_{13}-\frac{1}{2} c_{23}\right)
$$

(using (2.6)-(2.8)), hence

$$
\begin{aligned}
& y(m y)=\lambda^{2}\left(\alpha_{1} \varepsilon_{1}+\alpha_{2} \varepsilon_{2}+4 \alpha_{3} \varepsilon_{3}+a_{12}+\frac{1}{4} b_{13}+\frac{1}{4} c_{23}\right), \\
& m(y y)=\lambda^{2}\left(\alpha_{1} \varepsilon_{1}+\alpha_{2} \varepsilon_{2}+4 \alpha_{3} \varepsilon_{3}+a_{12}+\frac{5}{2} b_{13}+\frac{5}{2} c_{23}\right),
\end{aligned}
$$

and therefore

$$
-\langle m, y\rangle(y)=\lambda^{2}\left(-\frac{9}{4}\right)\left(b_{13}+c_{23}\right)=\mu^{2} m .
$$

It follows that $(-9 / 4) \lambda^{2}=\mu^{2}$, and therefore that $\mu= \pm 2 \pi i$. The eigenvalues of exp ad $z$ are obtained by exponentiating, hence 6.5(a) is proved.

If $(\operatorname{ad} z)(A e \otimes m)=\mu A e \otimes m, 0 \neq A \in C, 0 \neq m \in M_{3}^{8}$, then

$$
i A e \otimes m y=\mu A e \otimes m,
$$

and by writing $m$ in the form of (2.3), and using (2.6)-(2.8) as above, we obtain 


$$
\begin{aligned}
i A \lambda\left(\alpha_{1} \varepsilon_{1}\right. & \left.+\alpha_{2} \varepsilon_{2}-2 \alpha_{3} \varepsilon_{3}+a_{12}-\frac{1}{2} b_{13}-\frac{1}{2} c_{23}\right) \\
& =\mu A\left(\alpha_{1} \varepsilon_{1}+\alpha_{2} \varepsilon_{2}+\alpha_{3} \varepsilon_{3}+a_{12}+b_{13}+c_{23}\right) .
\end{aligned}
$$

Since one of $\alpha_{i}, a, b, c \neq 0$, we have either

$$
i \lambda=\mu, \quad-2 i \lambda=\mu \quad \text { or }-\frac{1}{2} i \lambda=\mu,
$$

hence $\mu=4 \pi i / 3,-8 \pi i / 3$ or $-2 \pi i / 3$. This proves $6.5(\mathrm{~b})$

Part (c) of 6.5 is proved in the same way.

Next we observe that the subspaces (6.4) are invariant under elements of $\operatorname{ad}_{\mathscr{E}_{7}} \mathscr{E}_{6}$. This follows immediately from (2.13) and (2.14). Thus the transformation ad $(d+(i h / 2) \otimes m), d \in \mathscr{F}_{4}, m \in M_{3}^{8}(0)$, has matrix representation

$$
\left[\begin{array}{lll}
A & 0 & 0 \\
0 & B & 0 \\
0 & 0 & C
\end{array}\right],
$$

where $A, B, C$ are square matrices representing the action of $\operatorname{ad}(d+(i h / 2) \otimes m)$ on the subspaces (6.4). Thus exp ad $(d+(\mathrm{ih} / 2) \otimes m)$ has matrix

$$
\left[\begin{array}{ccc}
\exp A & 0 & 0 \\
0 & \exp B & 0 \\
0 & 0 & \exp C
\end{array}\right]
$$

and from 6.5, exp ad $z$ commutes with all such elements of $E_{7}^{\prime}$, i.e. with all elements of $E_{6}^{\prime}$. Thus exp ad $z \in Z\left(E_{6}^{\prime}\right)$, is not the identity, and has order 3. This completes the proof of 6.3 .

Finally if $E_{7}^{\prime}$ is not simply connected, the inclusion $E_{6}^{\prime} \subset E_{7}^{\prime}$ can be lifted to an inclusion $E_{6}^{\prime} \subset E_{7}$, where $E_{7}$ is the simply connected form. We obtain

$$
F_{4} \subset E_{6}^{\prime} \approx E_{6} \subset E_{7} \text {. }
$$

This, together with the remarks at the beginning of this section, yields the proof of 6.1, hence the isomorphisms (1.4) are established.

7. Proof of Theorem 1.6. In this section we prove Theorem 1.6 by making use of É. Cartan's classification of symmetric spaces. To begin, we recall that Harris [8] has proved this result for the following pairs $(G, K)$ :

$$
\begin{aligned}
\left(\mathrm{SU}_{2 n+1}, \mathrm{SO}_{2 n+1}\right) & \text { with } L=\mathrm{Sp}_{2 n+1}, \\
\left(\mathrm{SU}_{2 n}, \mathrm{Sp}_{n}\right) \text { with } L & =\mathrm{SO}_{4 n}, \\
\left(\mathrm{Spin}_{2 n}, \mathrm{Spin}_{2 n-1}\right) \text { with } L & =\mathrm{Spin}_{2 n+1},
\end{aligned}
$$

and by the main result (1.4) of the last section, the theorem holds for the pair

$$
\left(E_{6}, F_{4}\right) \text { with } L=E_{7} \text {. }
$$


The fact that in each of the above cases the inequality rank $L-\operatorname{rank} G \leqq 1$ holds is easily checked. Moreover,

$$
\begin{array}{ll}
\mathrm{Sp}_{2 n+1} / U_{2 n+1}, & \mathrm{SO}_{4 n} / U_{2 n}, \\
\mathrm{Spin}_{2 n+1} / \operatorname{Spin}_{2 n}, & E_{7} / E_{6} \times S^{1},
\end{array}
$$

are symmetric spaces, and $U_{k} \approx \mathrm{SU}_{k} \times S^{1}$, hence in each of these cases $L / G$ or $L / M$ is a symmetric space, where $M$ is locally isomorphic to $G \times S^{1}$. This shows that (1.6) is satisfied for these pairs $(G, K)$.

Now the pairs $(G, K)$, where $K$ is the identity component of the fixed point set of an involution $\sigma$ of $C$, are classified by É. Cartan (see Helgason [9, p. 354]). In addition to those mentioned above, we have the pairs $(G, K)$ listed in the following table (7.1). (We drop the assumption of simple connectedness for the purposes of the table.)

TABLE 7.1

\begin{tabular}{llllll} 
& \multicolumn{1}{r}{$G$} & \multicolumn{1}{r}{$K$} & \multicolumn{1}{r}{ rank $G$} & \multicolumn{1}{r}{ rank $K$} & \\
\hline 1 & $\mathrm{SU}_{2 n}$ & $\mathrm{SO}_{2 n}$ & $2 n-1$ & $n$ & \\
2 & $\mathrm{SU}_{p+q}$ & $S\left(U_{p} \times U_{q}\right)$ & $p+q-1$ & $p+q-1$ & \\
3 & $\mathrm{SO}_{p+q}$ & $\mathrm{SO}_{p} \times \mathrm{SO}_{q}$ & $r+s$ & $r+s$ & $(p=2 r, q=2 s)$ \\
4 & & & $r+s$ & $r+s$ & $(p=2 r, q=2 s+1)$ \\
5 & & & $r+s$ & $r+s$ & $(p=2 r+1, q=2 s)$ \\
6 & & & $r+s+1$ & $r+s$ & $(p=2 r+1, q=2 s+1)$ \\
7 & $\mathrm{SO}_{2 n}$ & $U_{n}$ & $n$ & $n$ & \\
8 & $\mathrm{Sp}_{n}$ & $U_{n}$ & $n$ & $n$ & \\
9 & $\mathrm{Sp}_{p+q}$ & $\mathrm{Sp}_{p} \times \mathrm{Sp}_{q}$ & $p+q$ & $p+q$ & \\
10 & $E_{6}$ & $\mathrm{Sp}_{4}$ & 6 & 4 & \\
11 & $E_{6}$ & $\mathrm{SU}_{6} \times \mathrm{SU}_{2}$ & 6 & $5+1$ & \\
12 & $E_{6}$ & $\mathrm{SO}_{10} \times S^{1}$ & 6 & $5+1$ & \\
13 & $E_{7}$ & $\mathrm{SU}_{8}$ & 7 & 7 & \\
14 & $E_{7}$ & $\mathrm{SO}_{12} \times \mathrm{SU}_{2}$ & 7 & $6+1$ & \\
15 & $E_{7}$ & $E_{6} \times S^{1}$ & 7 & $6+1$ & \\
16 & $E_{8}$ & $\mathrm{SO}_{16}$ & 8 & 8 & \\
17 & $E_{8}$ & $E_{7} \times \mathrm{SU}_{2}$ & 8 & $7+1$ & \\
18 & $F_{4}$ & $\mathrm{Sp}_{3} \times \mathrm{SU}_{2}$ & 4 & $3+1$ & \\
19 & $F_{4}$ & $\mathrm{SO}_{9}$ & 4 & 4 & \\
20 & $G_{2}$ & $\mathrm{SU}_{2} \times \mathrm{SU}_{2}$ & 2 & $1+1$ &
\end{tabular}

Observe that the case ( $\left.\operatorname{Spin}_{2 n}, \operatorname{Spin}_{2 n-1}\right)$ is listed as type 6 in the table where $r=n-1, s=0, \quad($ hence $p+q=(2 r+1)+(2 s+1)=2(n-1)+1+1=2 n)$, and $\mathrm{SO}_{1}$ is a point. 
Proposition 7.2. The only pairs $(G, K)$ satisfying the hypotheses of Theorem 1.6 are:

$$
\left(\mathrm{SU}_{2 n+1}, \mathrm{SO}_{2 n+1}\right), \quad\left(\mathrm{SU}_{2 n}, \mathrm{Sp}_{n}\right), \quad\left(\operatorname{Spin}_{2 n}, \operatorname{Spin}_{2 n-1}\right), \quad\left(E_{6}, F_{4}\right) .
$$

To prove this we use the following:

LEMMA 7.3. If $K$ has maximal rank in $G$, then $H^{*}(G ; R) \rightarrow H^{*}(K ; R)$ induced by inclusion, is not an epimorphism.

Proof of 7.3. If $K$ has maximal rank in $G$, the Euler-Poincaré characteristic $\chi(G / K)$ is positive. (See Hopf-Samelson [11].) If $H^{*}(G ; R) \rightarrow H^{*}(K ; R)$ is onto, then by Harris' result (Theorem 5.1(c)), $G / K$ would have the homology of a product of odd spheres. Since $\chi($ odd sphere $)=0, \chi(G / K)=0$, a contradiction.

In the table (7.1) we have noted the ranks of $G$ and $K$. All but the following pairs are therefore eliminated by (7.3):

(a) $\left(\mathrm{SU}_{2 n}, \mathrm{SO}_{2 n}\right)$;

(b) $\left(\mathrm{SO}_{2 r+1+2 s+1}, \mathrm{SO}_{2 r+1} \times \mathrm{SO}_{2 s+1}\right) \quad(r, s \neq 0)$;

(c) $\left(E_{6}, \mathrm{Sp}_{4}\right)$.

To complete the proof of (7.2) we show that in each of the cases (a)-(c), the map $H^{*}(G ; R) \rightarrow H^{*}(K ; R)$ is not an epimorphism. (Equivalently, $H_{*}(K ; R) \rightarrow H_{*}(G ; R)$ is not 1-1.)

The real cohomology of the simple groups is known (see Borel [3], Borel and Chevalley [4]).

Recall that $H^{*}\left(E_{6} ; R\right)=\Lambda\left(x_{3}, x_{9}, x_{11}, x_{15}, x_{17}, x_{23}\right)$ and $H_{*}\left(\operatorname{Sp}_{4} ; R\right)$ $=\Lambda\left(x_{3}, x_{7}, x_{11}, x_{15}\right)$, hence the inclusion $\mathrm{Sp}_{4} \subset E_{6}$ cannot be 1-1 in homology, for $H^{*}\left(E_{6} ; R\right)$ contains nothing in degree 7. This eliminates (c).

Similarly, if $r, s \neq 0$,

$$
\begin{aligned}
H_{*}\left(\mathrm{SO}_{2(r+s+1)} ; R\right) & =\Lambda\left(x_{3}, x_{7}, \cdots, x_{4(r+s+1)-5}, Y_{2(r+s+1)-1}\right), \\
H_{*}\left(\mathrm{SO}_{2 r+1} ; R\right) & =\Lambda\left(x_{3}, x_{7}, \cdots, x_{4 r-1}\right),
\end{aligned}
$$

hence $H_{*}\left(\mathrm{SO}_{2 r+1} \times \mathrm{SO}_{2 s+1} ; R\right)$ has two independent generators in degree 3 . Thus the inclusion $\mathrm{SO}_{2 r+1} \times \mathrm{SO}_{2 s+1} \rightarrow \mathrm{SO}_{2(r+s+1)}$ is not 1-1 in homology. This eliminates (b).

Finally, recall that $H_{*}\left(\mathrm{SU}_{2 n} ; R\right)=\Lambda\left(x_{3}, x_{5}, \cdots, x_{4 n-1}\right)$ and $H_{*}\left(\mathrm{SO}_{2 n} ; R\right)$ $=\Lambda\left(w_{3}, w_{7}, \cdots, w_{4 n-5}, y_{2 n-1}\right)$. Let $\sigma$ be the automorphism of $\mathrm{SU}_{2 n}$ with $\mathrm{SO}_{2 n}$ as fixed point set.

If $n=2 k$, then $H_{*}\left(\mathrm{SO}_{2 n} ; R\right)$ has two generators of degree $2 n-1=4 k-1$. Thus, the inclusion $i: \mathrm{SO}_{2 n} \rightarrow \mathrm{SU}_{2 n}$ is not 1-1 in homology.

If $n=2 k+1$, and $i^{*}: H^{*}\left(\mathrm{SU}_{2 n} ; R\right) \rightarrow H^{*}\left(\mathrm{SO}_{2 n} ; R\right)$ is an epimorphism, then by Harris' result (5.1), $H^{*}\left(\mathrm{SU}_{2 .:} ; R\right) \approx U \otimes V, U$ the subalgebra generated by those $x_{i}$ such that $\sigma^{*}\left(x_{i}\right)=x_{i}$, and $V$ the subalgebra generated by those $x_{i}$ such that $\sigma^{*}\left(x_{i}\right)=-x_{i}$. Moreover, $i^{*}$ is zero on the positive degree elements of $V$ and maps $U$ isomorphically onto $H^{*}\left(\mathrm{SO}_{2 n} ; R\right)$. 
From Jacobson [12, p. 281], $A\left(\mathrm{SU}_{2 n}\right) / I\left(\mathrm{SU}_{2 n}\right)=Z_{2}$, and $\sigma$ is of order 2 and not inner. Hence (5.2) applies to $\mathrm{SU}_{2 n}$, and we see that if $\operatorname{deg} x_{i}=4 k_{i}+1$, then $\sigma^{*}\left(x_{i}\right)=-x_{i}$.

Now deg $y_{2 n-1}=4 k+1$, and $i^{*}$ is onto, so $y_{4 k+1}=y_{2 n-1}=i^{*}\left(t x_{4 k+1}+d\right)$, $t \in R, d$ decomposable. Since $i^{*}(d)=y_{4 k+1}-t i^{*}\left(x_{4 k+1}\right)$ is primitive and decomposable, we have $i^{*}(d)=0$. Thus $i^{*}\left(t x_{4 k+1}\right)=y_{4 k+1}$. But $\sigma^{*}\left(x_{4 k+1}\right)=-x_{4 k+1}$, by the remark of the preceding paragraph, hence

$$
\sigma^{*}\left(y_{4 k+1}\right)=\sigma^{*} i^{*}\left(t x_{4 k+1}\right)=i^{*} \sigma^{*}\left(t x_{4 k+1}\right)=-i^{*}\left(t x_{4 k+1}\right)=-y_{4 k+1},
$$

a contradiction, since $\sigma^{*}$ is the identity on $H^{*}\left(\mathrm{SO}_{2 n}: R\right)$. Thus $i^{*}$ is not onto, and case (a) is eliminated.

The proof of Theorem 1.6 is therefore complete.

\title{
REFERENCES
}

1. S. Araki, On the Brouwer degrees of some maps of compact symmetric spaces, Topology Vol. 3, Pergamon, New York, 1965, pp. 281-290.

2. E. Artin, Geometric algebra, Interscience Tract No. 3, Wiley, New York, 1957.

3. A. Borel, Sur la cohomologie des espaces fibrés principaux et des espaces homogènes de groupes de Lie compacts, Ann. of Math. 57 (1953), 115-207.

4. A. Borel and C. Chevalley, The Betti numbers of the exceptional groups, Mem. Amer. Math. Soc. No. 14 (1955), 1-9.

5. A. Borel and J. de Siebenthal, Les sous-groupes fermés connexes de rang maximum des groupes de Lie clos, Comment. Math. Helv. 23 (1949-1950), 200-221.

6. R. Bott, The stable homotopy of the classical groups, Ann. of Math. 70 (1959), 313-337.

7. E. B. Dynkin and A. L. Oniščik, Compact global Lie groups, Uspehi. Mat. Nauk (N.S.) 10 (1955), 3-74; English transl., Amer. Math. Soc. Transl. (2) 21 (1962), 119-192.

8. B. Harris, Suspensions and characteristic maps for symmetric spaces, Ann. of Math. 76 (1962), 295-305.

9. S. Helgason, Differential geometry and symmetric spaces, Academic Press, New York, 1962.

10. P. Hilton and S. Wiley, Homology theory, Cambridge Univ. Press, New York, 1960.

11. H. Hopf and H. Samelson, Ein Satz über die Wirkungsräume geschlossener Leischer Gruppen, Comment. Math. Helv. 13 (1940-1941), 240-251.

12. N. Jacobson, Lie algebras, Interscience Tract No. 10, Wiley, New York, 1962.

13. —_ Some groups of transformations defined by Jordan algebras. I, II, III, J. Reine Angew. Math. I: 201 (1959), 178-195, II: 204 (1960), 74-98, III: 207 (1961), 61-85.

14. J.-P. Serre, Groupes d'homotopie et classes de groupes abéliens, Ann. of Math. 58 (1953), 258-294.

15. N. E. Steenrod, The topology of fibre bundles, Princeton Univ. Press, Princeton, N. J., 1951.

16. J. Tits, Une classe d'algebres de Lie en relation avec les algebres de Jordan, Nederl. Akad. Wetensch. Proc. Ser. A 65 (1962), 530-535.

\author{
BROWN UNIVERSITY, \\ Providence, RHodb IsLaNd \\ State University of New York at Stony Brook, \\ STONY BROOK, NEW YORK
}

